\title{
ANÁLISE QUALI-QUANTITATIVA DA ARBORIZAÇÃO DE RUAS DO MUNICÍPIO DE GODOY MOREIRA - PR
}

\author{
QUALI-QUANTITATIVE EVALUATION OF THE URBAN AFFORESTATION OF \\ GODOY MOREIRA - PR
}

Yara Campos Miranda ${ }^{1}$, Mariana de Sarges Machado ${ }^{1}$, Lorene dos Santos Silva ${ }^{1}$, Renata Estevam¹, Francisco Ferreira Martins Neto ${ }^{2}$, Marcelo Galeazzi Caxambu ${ }^{3}$

\section{RESUMO}

Arborização urbana pode ser considerada como fator determinante de salubridade ambiental, sendo constituída por toda cobertura vegetal de porte arbóreo existente nas cidades. A importância do planejamento correto evita plantios irregulares, obstrução da viabilidade urbana e, principalmente, a introdução de espécies exóticas e invasoras. Em muitos casos esse planejamento arbóreo é ausente, ocasionando problemas ao longo dos anos nas espécies plantadas. O trabalho teve como finalidade avaliar a arborização de acompanhamento viário de Godoy Moreira-PR. As coletas de material botânico foram conduzidas de acordo com as técnicas usuais em levantamentos florísticos e a identificação ocorreu no Herbário da Universidade Tecnológica Federal do Paraná Campus Campo Mourão (HCF). Adicionalmente, as espécies foram classificadas como nativas, exóticas e exóticas invasoras, sendo estas últimas estabelecidas com base na legislação Paranaense. Foram encontrados 649 indivíduos, distribuídos em 27 famílias botânicas e 47 espécies, sendo 24\% nativas e $76 \%$ exóticas. As maiores frequências apresentadas foram em Poincianella pluviosa com $28,96 \%$, Terminalia catappa $16,46 \%$ e Licania tomentosa 10,76\%, representando $56 \%$ da arborização urbana do Município. Estas espécies são conflitantes com a urbe, pois causam danos ao calçamento e a rede elétrica, além de produzirem grande quantidade de biomassa, o que está em desacordo com a norma de acessibilidade urbana.

Palavras-chave: Espécies invasoras; Espécies exóticas; Poincianella pluviosa.

\section{ABSTRACT}

Urban afforestation can be considered as a determinant factor of the environmental salubrity, consisting of all plant coverage existent in the urban area. The importance of a correct planning is to avoid irregular planting of trees, obstruction of urban spaces and, most important, to avoid the introduction of exotic and invasive plants. In most of the cases, the arboreal planning is not present, leading to problems over the years related with those species of trees planted. In this respect, the present study intended to evaluate the urban afforestation in sidewalks of Godoy Moreira-PR. The collection of the botanical materials in were conducted according to the usual techniques adopted in floristics inventories and the identification performed at the Herbarium of Universidade Tecnológica Federal do Paraná (HCF) located in Campo Mourão. In addition, the collected species were classified as native, exotic and exotic invasive, being the last two classified according to the Paraná's legislation. The total quantification resulted in 649 samples, classified in 27 botanical families and 47 species, being $24 \%$ native and $76 \%$ exotic species. The majority of species sampled were Poincianella pluviosa with $28.96 \%$, Terminalia catappa with $16.46 \%$ and Licania tomentosa with $10,76 \%$, representing $56 \%$ of all plant species present in the city. These species are urban problematic because they cause damages to sidewalks and the electric network, moreover they produce great amounts of biomass, which are not in accordance to the urban accessibility norms.

Key-words: Invasive species; Exotic species; Poincianella pluviosa.

\footnotetext{
Recebido em 14.07.2015 e aceito em 15.09.2015

1 Graduanda em Engenharia Ambiental, Universidade Tecnológica Federal do Paraná (UTFPR), Campo Mourão/PR. Email: yaracamposmiranda1@gmail.com; mari_msm@hotmail.com; lorenesantosdasilva@gmail.com; renata.engenhariacm@gmail.com 2 Graduando em Engenharia Ambiental, UTFPR, Campo Mourão/PR. Email: neto.fm@hotmail.com

3 Engenheiro Florestal, Universidade Tecnológica Federal do Paraná, Campo Mourão/PR. Email: mcaxambu@utfpr.edu.br
} 


\section{INTRODUÇÃO}

Nos últimos anos, devido ao processo intenso de urbanização, onde há uma tendência mundial de concentração da população nas áreas urbanas, muitas cidades crescem de maneira exacerbada e sem planejamento, o que ocasiona danos ambientais, refletindo na qualidade de vida da população. Uma forma de mitigar alguns problemas causados por este processo, é a arborização urbana, que proporciona conforto térmico, diminuição dos efeitos da poluição, redução do impacto da chuva sobre o solo, além da absorção de parte dos raios solares, sendo fundamental para a melhoria da qualidade de vida (EMER et al., 2014).

Apesar de todos os benefícios, muitas vezes o planejamento urbano não considera as importantes técnicas de manejo e planejamento da arborização de ruas, sugerindo espécies que causam transtornos aos munícipes, bem como à estrutura das vias e calçadas. O nível de toxicidade da planta e sua capacidade em se sobressair sobre as demais espécies, se tornando uma ameaça à biodiversidade local, também devem ser considerados. Para tanto, as normas de acessibilidade e as portarias que alertam para o plantio de espécies exóticas devem ser observadas e seguidas com o máximo critério (KÜSTER et al., 2012).

De acordo com a COPEL (2009) as espécies indicadas para arborização urbana devem, preferencialmente, ser nativas da região, possuir resistência a pragas e doenças, apresentar velocidade de crescimento média/rápida, possuir troncos resistentes para evitar a queda, dispor de folhagem com renovação e tamanho favoráveis, para não entupir canalizações com a queda de folhas e apresentar copas adequadas para não prejudicar a fiação elétrica e a passagem de pedestres.

Iniciativas particulares e sem o conhecimento técnico adequado, ainda são as maiores responsáveis pelo plantio inadequado de espécies na arborização urbana de vias públicas, ressaltam Biondi e Pedrosa-Macedo (2007). Essa falta de planejamento acarreta altos custos para o município, tornando a arborização um problema urbano de grandes proporções.

Nessa perspectiva, o presente trabalho teve como finalidade avaliar qualiquantitativamente a arborização de ruas e avenidas que compõem o acompanhamento viário do Município de Godoy Moreira. Vale ressaltar que a área de estudo não possui trabalhos referentes a arborização e planejamento urbano, sendo que estes dados poderão compor o plano de arborização urbana do município. 


\section{MATERIAL E MÉTODOS}

\section{Caracterização da área de estudo}

Este estudo foi conduzido no município de Godoy Moreira, região central do estado do Paraná que possui uma população estimada de 3.337 habitantes (Figura 1). Suas

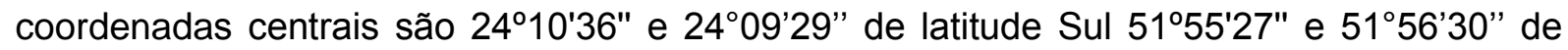
longitude Oeste, com altitude média de 512 metros em relação ao nível do mar. O município se desmembrou de São João do Ivaí tendo limites de fronteiras com as seguintes cidades: Barbosa Ferraz, São João do Ivaí, Lunardelli, Jardim Alegre e Iretama (IBGE, 2014).

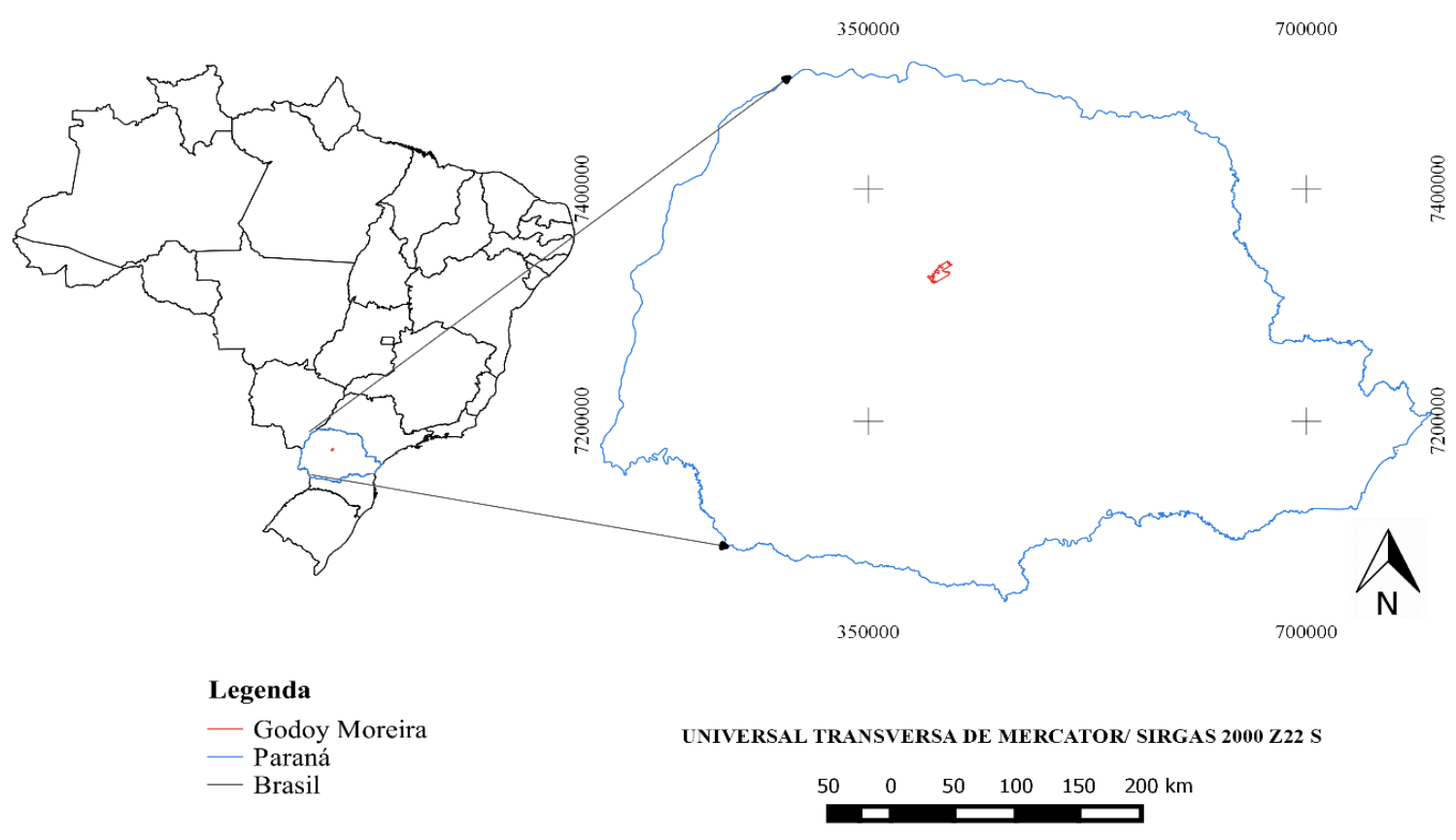

Figura 1. Localização do Município de Godoy Moreira no Estado do Paraná e no Brasil Figure 1. The location of Godoy Moreira in the state of Parana and in Brazil

O município de Godoy Moreira, de acordo com a classificação de Koppen-Geiger em Cfa - clima subtropical, com temperatura média no período de verão superior a $22{ }^{\circ} \mathrm{C} \mathrm{e}$ temperatura média no inverno inferior a $18^{\circ} \mathrm{C}$ (CAVIGLIONE, 2000).

Possui predominância de Neossolo Litólico, Nitossolo Vermelho e Latossolo Vermelho associados a um relevo levemente ondulado a ondulado (EMBRAPA, 2007).

Quanto a vegetação, Godoy Moreira pertence ao Bioma Mata Atlântica, que de acordo com a classificação proposta por Roderjan et. al. (2002), cuja formação florestal é, em essência, a floresta Estacional Semidecidual. 


\section{Levantamento quantitativo}

No censo arbóreo do município de Godoy Moreira foi realizada análise qualitativa e quantitativa, sendo inclusas todas as espécies com DAP acima de 0,4 m, e com porte arbóreo, englobando neste caso as árvores, palmeiras e arbustos conduzidos através de podas, presentes na zona urbana do município, acompanhando suas ruas e avenidas.

Indivíduos arbóreos, palmeiras e arbustos conduzidos são amplamente utilizados em diversas cidades paranaenses, e compõem de maneira significativa a arborização urbana, devido a esse fato, foram inclusos no censo.

No dia 7 de abril de 2015, foi conduzida a coleta do material arbóreo e posteriormente o mesmo foi herborizado, de acordo com as técnicas descritas em IBGE (2012). Posteriormente, as espécies foram identificadas no Herbário da Universidade Tecnológica Federal do Paraná Campus Campo Mourão (HCF), através de comparação com material previamente armazenado e por especialistas. As famílias foram classificadas de acordo com Lista da Flora do Brasil (2015) e APG III (2009).

\section{Levantamento qualitativo}

Para análise qualitativa, as espécies obtidas através do censo realizado, foram classificadas em exóticas invasoras, subdividas em duas categorias, Exóticas Invasoras Categoria I (El1) e Exóticas Invasoras Categoria II (EI2) de acordo com a Portaria IAP 59/2015 (lista oficial de espécies exóticas invasoras para o estado do Paraná). A classificação em espécies nativas, exóticas e exóticas no Paraná baseou-se na Lista de Espécies da Flora do Brasil (2015). Assim, foram definidas as seguintes categorias:

- Nativa $(\mathrm{N})$ : espécie ocorrente dentro de sua área de distribuição natural presente ou passada;

- Exóticas (E): as espécies introduzidas fora de sua área natural de distribuição;

- Exóticas Invasoras Categoria I (El1): espécies invasoras que não devem ser cultivadas ou criadas ficando seu uso em qualquer uma das formas não permitidas;

- Exóticas Invasoras Categoria II (EI 2): espécies utilizadas em sistema de produção e com valor comercial, que podem ser criadas ou cultivadas em condições controladas sob regulamentação específica;

- Exóticas no Estado do Paraná (Ex-PR) espécies que ocorrem no território nacional porém não tem sua distribuição natural no estado do Paraná. 


\section{RESULTADOS E DISCUSSÃO}

No censo arbóreo realizado no Município de Godoy Moreira - PR foram encontrados 632 indivíduos, plantados nas ruas e avenidas da urbe, distribuídos em 45 espécies e 26 famílias botânicas (tabela 1). As espécies com maior abundância foram Poincianella pluviosa var. peltophoroides (Benth.) L.P.Queiroz (sibipiruna), Terminalia catappa L. (amendoeira) e Licania tomentosa (Benth.) Fritsch (oiti), que somam 355 exemplares, representando $56 \%$ da cobertura arbórea da cidade.

Tabela 1. Espécies presentes na arborização urbana do município de Godoy Moreira - PR Table 1. List of species present in the urban area of Godoy Moreira - PR

\begin{tabular}{|c|c|c|c|c|}
\hline Família & Família/ Nome cientifico & Origem & Quantidade & $\begin{array}{l}\text { Frequência } \\
(\%)\end{array}$ \\
\hline \multirow{2}{*}{ ANACARDIACEAE } & Mangifera indica L. & EI 2 & 21 & 3,32 \\
\hline & Schinus molle L. & $\mathrm{N}$ & 20 & 3,16 \\
\hline ANNONACEAE & Annona muricata L & $E$ & 3 & 0,47 \\
\hline APOCYNACEAE & Nerium oleander $\mathrm{L}$. & $E$ & 12 & 1,90 \\
\hline \multirow{4}{*}{ ARECACEAE } & $\begin{array}{c}\text { Cocos nucifera L. } \\
\text { Dvpsis lutescens }(\mathrm{H} . \text { Wendl.) }\end{array}$ & Ex PR & 2 & 0,32 \\
\hline & Beentje \& J. Dransf & $E$ & 4 & 0,63 \\
\hline & $\begin{array}{c}\text { Euterpe edulis Mart. } \\
\text { Livistona chinensis (Jacq.) }\end{array}$ & $\mathrm{N}$ & 11 & 1,74 \\
\hline & R.Br. ex Mart. & $E$ & 1 & 0,16 \\
\hline \multirow{3}{*}{ BIGNONIACEAE } & $\begin{array}{l}\text { Handroanthus chrysotrichus } \\
\text { (Mart. ex DC.) Mattos } \\
\text { Handroanthus heptaphyllus }\end{array}$ & $\mathrm{N}$ & 5 & 0,79 \\
\hline & $\begin{array}{c}\text { (Vell.) Mattos } \\
\text { Spathodea campanulata P. }\end{array}$ & $\mathrm{N}$ & 37 & 5,85 \\
\hline & Beauv. & $E$ & 2 & 0,32 \\
\hline CACTACEAE & $\begin{array}{c}\text { Pereskia grandiflora Haw. I } \\
\text { Pfeiff. }\end{array}$ & $\mathrm{N}$ & 5 & 0,79 \\
\hline CHRYSOBALANACEAE & $\begin{array}{c}\text { Licania tomentosa (Benth.) } \\
\text { Fritsch }\end{array}$ & Ex PR & 68 & 10,76 \\
\hline COMBRETACEAE & Terminalia catappa L. & $\mathrm{El} 2$ & 104 & 16,46 \\
\hline \multirow{8}{*}{ FABACEAE } & $\begin{array}{c}\text { Bauhinia variegata } \mathrm{L} . \\
\text { Caesalpinia pulcherrima (L.) }\end{array}$ & $E$ & 6 & 0,95 \\
\hline & & $E$ & 4 & 0,63 \\
\hline & $\begin{array}{c}\text { Cassia fistula L. } \\
\text { Clitoria fairchildiana R.A. }\end{array}$ & $E$ & 4 & 0,63 \\
\hline & $\begin{array}{c}\text { Howard } \\
\text { Delonix regia (Bojer ex Hook.) }\end{array}$ & $\mathrm{N}$ & 1 & 0,16 \\
\hline & $\begin{array}{c}\text { Raf. } \\
\text {. }\end{array}$ & $E$ & 13 & 2,06 \\
\hline & $\begin{array}{l}\text { de Wit } \\
\text { Poincianella pluviosa var. } \\
\text { peltophoroides (Benth.) }\end{array}$ & El 1 & 7 & 1,11 \\
\hline & L.P.Queiroz & Ex PR & 183 & 28,96 \\
\hline & Pterogyne nitens Tul. & $\mathrm{N}$ & 1 & 0,16 \\
\hline LAURACEAE & Persea americana Mill. & $E$ & 2 & 0,32 \\
\hline
\end{tabular}




\begin{tabular}{|c|c|c|c|c|}
\hline Família & Família/ Nome cientifico & Origem & Quantidade & \begin{tabular}{|c|}
$\begin{array}{c}\text { Frequência } \\
(\%)\end{array}$ \\
\end{tabular} \\
\hline LYRTACEAE & Punica granatum $\mathrm{L}$. & $E$ & 1 & 0,16 \\
\hline MAGNOLIACEAE & $\begin{array}{c}\text { Magnolia champaca (L.) Baill. } \\
\text { ex Pierre }\end{array}$ & El 2 & 2 & 0,32 \\
\hline MALPHIGUIACEAE & Malpighia emarginata DC & $E$ & 5 & 0,79 \\
\hline MALVACEAE & $\begin{array}{l}\text { Hibiscus rosa-sinensis L. } \\
\text { Pachira aquatica Aubl. }\end{array}$ & $\begin{array}{l}E \\
N\end{array}$ & $\begin{array}{l}3 \\
5\end{array}$ & $\begin{array}{l}0,47 \\
0,79\end{array}$ \\
\hline MELASTOMATACEAE & $\begin{array}{c}\text { Tibouchina granulosa (Desr.) } \\
\text { Cogn. }\end{array}$ & $\mathrm{N}$ & 4 & 0,63 \\
\hline MELIACEAE & $\begin{array}{l}\text { Cedrela fissilis Vell. } \\
\text { Melia azedarach L. }\end{array}$ & $\begin{array}{c}\mathrm{N} \\
\mathrm{EI} 1\end{array}$ & $\begin{array}{l}9 \\
8\end{array}$ & $\begin{array}{l}1,42 \\
1,27\end{array}$ \\
\hline MORACEAE & $\begin{array}{l}\text { Artocarpus heterophyllus Lam. } \\
\text { Ficus benjamina L. }\end{array}$ & $\begin{array}{l}E \\
E\end{array}$ & $\begin{array}{c}1 \\
17 \\
\end{array}$ & $\begin{array}{l}0,16 \\
2,69\end{array}$ \\
\hline MYRTACEAE & $\begin{array}{c}\text { Eugenia uniflora L. } \\
\text { Psidium guajava L. } \\
\text { Syzygium jambos (L.) Alston }\end{array}$ & $\begin{array}{l}\mathrm{N} \\
\mathrm{El} 2 \\
\mathrm{El} 2 \\
\end{array}$ & $\begin{array}{l}3 \\
6 \\
9\end{array}$ & $\begin{array}{l}0,47 \\
0,95 \\
1,42 \\
\end{array}$ \\
\hline OLEACEAE & Ligustrum lucidum W.T. Aiton & El 1 & 9 & 1,42 \\
\hline PINACEAE & Pinus taeda L. & $\mathrm{El} 2$ & 1 & 0,16 \\
\hline ROSACEAE & $\begin{array}{c}\text { Eriobotrya japonica (Thunb.) } \\
\text { Lindl.bio }\end{array}$ & El 2 & 9 & 1,42 \\
\hline RUTACEAE & $\begin{array}{c}\text { Citrus sp. } \\
\text { Citrus deliciosa tem. } \\
\text { Murraya paniculata (L.) Jack }\end{array}$ & $\begin{array}{c}E \\
E \\
\text { EI } 1\end{array}$ & $\begin{array}{c}1 \\
7 \\
10\end{array}$ & $\begin{array}{l}0,16 \\
1,11 \\
1,58\end{array}$ \\
\hline SAPOTACEAE & $\begin{array}{l}\text { Mimusops commersonii (G. } \\
\text { Don) Engl. }\end{array}$ & $E$ & 1 & 0,16 \\
\hline SOLANACEAE & $\begin{array}{c}\text { Brunfelsia uniflora (Pohl) D. } \\
\text { Don }\end{array}$ & $\mathrm{N}$ & 2 & 0,32 \\
\hline VERBENACEAE & Duranta vestita Cham. & $\mathrm{N}$ & 3 & 0,47 \\
\hline
\end{tabular}

Ao se considerar as espécies registradas neste estudo, de acordo com sua origem, é notável que a maioria, cerca de $76 \%$ delas são exóticas ou exóticas invasoras. A portaria IAP n 59 de 2015 reconhece as espécies exóticas invasoras no estado do Paraná e as classifica de acordo com seu potencial invasor. Nesta condição estão Leucaena leucocephala (Lam.) de Wit (leucena), Melia azedarach L. (santa-bárbara), Ligustrum lucidum W.T. Aiton (Ligustro), Murraya paniculata (L.) Jack (murta), dispostas na categoria I da Portaria 59 de 2015, representando 12\% dos indivíduos classificados como exóticos. Ainda existe uma instrução normativa do IAP № 15.953/08 que proíbe o plantio da Murraya paniculata no estado do Paraná, por ser hospedeira da bactéria Candidatus liberibacter spp. disseminada pelo inseto vetor Diaphorina citri Kuwayama, 1908 (Hemiptera, Psyllidae), transmissor da praga denominada Huanglongbing (HLB - Greening), que ocasiona prejuízos à citricultura. 
$\mathrm{Na}$ categoria II, compondo $24 \%$ dos indivíduos amostrados como exóticos estão inclusas Mangifera indica L (mangueira), Magnolia champaca (L.) Baill. ex Pierre (magnólia), Psidium guajava L. (goiaba), Pinus taeda L. (pinheiro), Eriobotrya japonica (Thunb.) Lindl (ameixa-amarela) e Terminalia catappa.

O uso ou a permanência de espécies exóticas invasoras nas vias públicas deve ser evitado, haja visto que além de restrições normativas, segundo Blum, Borgo e Sampaio (2008) há o risco para a biodiversidade, uma vez que estas espécies podem competir e eliminar espécies nativas da região. Biondi e Pedrosa-Macedo (2007), alertam que a população e órgãos públicos são os principais responsáveis pela presença de exóticas invasoras na composição florística de uma cidade.

No levantamento realizado em Godoy Moreira foi possível constatar que $76 \%$ das espécies arbóreas estudadas eram exóticas ou exóticas invasoras. Este resultado se assemelha com o obtido por Blum, Borgo e Sampaio (2008), em Maringá - PR, em um levantamento de espécies exóticas, cujo número representou $72 \%$; em Souza e Paiva (2014) estudando bairros de São Paulo, foram 68\%; em Emer et al. (2014), na arborização dos bairros Veneza e Aeroporto na cidade de Pato Branco - PR, encontraram 58,2\%; em Biondi e Pedrosa-Macedo (2007), foram registradas 58,1\% de espécies exóticas ou exóticas invasoras em um levantamento realizado em Curitiba - PR. Em uma análise do acompanhamento viário do município de Farol- PR, Hoppen et al. (2014) relataram que 73\% das árvores da urbe eram exóticas para o Brasil.

Alguns municípios podem, por outro lado, apresentar um predomínio de espécies nativas, não se encaixando na situação relatada para Godoy Moreira, como em Maiorani et al. (2012) em um levantamento de arborização urbana de Altonia - PR e obtiveram 67\% de espécies nativas, Albertin et al. (2011) na arborização viária da cidade de Nova esperança PR, nas quais 68\% das espécies encontradas na composição arbórea eram nativas, tendendo assim a uma situação mais próxima da considerada por Blum, Borgo e Sampaio (2008) como ideal, onde as espécies exóticas invasoras devem ser substituídas gradualmente por nativas.

No entanto, algumas vezes, espécies nativas podem não estar adequadas a urbe, como por exemplo, a Poincianella pluviosa var. peltophoroides que foi a com maior frequencia amostrada, com cerca de $28 \%$, apresenta folhas pequenas e decíduas, que prejudicam a limpeza urbana e entopem bueiros. Embora nativa, esta espécie é exótica para o Estado do Paraná de acordo com a Flora do Brasil (2015). Este fato é preocupante quando se considera que a Copel (2009) recomenda que a frequência de dada espécie na arborização urbana não ultrapasse $10 \%$ a $15 \%$ do total ao se considerar a norma ISA. Outros estudos também demonstram que Poincianella pluviosa var peltophoroides é empregada na arborização de vias públicas de diversos municípios. Em Maringá Blum, 
Borgo e Sampaio (2008) em um levantamento de exóticas invasoras, registraram esta espécie como a de maior ocorrência, com 39\%. Valores consideráveis também foram alcançados por Martins et al. (2011) no acompanhamento viário do município de Luiziania PR, onde Poincianella pluviosa var. peltophoroides atingiu percentual de 16,9\%.

Outra espécie exótica encontrada em abundância no município de Godoy Moreira, representando $16 \%$ dos indivíduos, foi Terminalia catappa, considerada exótica invasora para o estado do Paraná de acordo com a Portaria IAP nำ59/2015, já citada anteriormente. Levantamentos realizados em diversas cidades também apontaram a espécie em questão como abundante, como Neto e Souza (2009) em um estudo das áreas verdes de Aracaju SE, Souza e Cintra (2007) na análise da arborização de acompanhamento viário realizado em Taquara - RJ, Iwata (2007) na análise florística do Bairro Vermelho em Teresina - PI. Nestes estudos, a frequência da espécie foi de respectivamente 20,3\%, 28,1\% e 37,4\%. Estes trabalhos demonstram que a Terminalia catappa está distribuída em todo o território nacional, e pode alcançar valores de frequência elevados

Terminalia catappa e Poincianella pluviosa var peltrophoroides não estão de acordo com o preconizado pela NBR 9050:2004 de acessibilidade, especificando que espécies adequadas na arborização urbana de vias públicas não podem produzir grande quantidade de biomassa por que prejudicam a limpeza da urbe, possuir raízes vigorosas ou porte elevado, podendo acarretar em conflitos com a rede elétrica além de ocasionar, frequentemente, danos a equipamentos públicos como calçadas e a malha viária.

Outras espécies foram encontradas, porém com menor frequência, algumas não são recomendadas por conterem potencial alergênico ou tóxico. De acordo com Souza et al. (2011), Kuster et al. (2012) e Cadorin et al. (2008) estas caraterísticas são encontradas, por exemplo, em Schinus molle L (chorão), Nerium oleander L. (espirradeira), Ligustrum lucidum e Melia azedarach.

O potencial de produção de biomassa, queda espontânea e alta produtividade de frutos, de acordo com observações in loco, foi registrado para Poincianella pluviosa var peltrophoroides, Ficus benjamina L. (figueira), Psidium guajava, Terminalia catappa, Delonix regia (Bojer ex Hook.) Raf (flamboyant), Mangifera indica Artocarpus heterophyllus Lam. (Jaqueira) Eriobotrya japonica e Citrus deliciosa Tem. (Laranjeira). Isto indica a necessidade de um manejo adequado para estas espécies consideradas problemáticas.

O presente trabalho reflete a inexistência de um plano de arborização urbana no município de Godoy Moreira. Este fato também foi observado em diferentes trabalhos, que observaram padrões semelhantes como Biondi e Pedrosa-Macedo (2007) em Curitiba - PR, Pagliari e Durigon (2013) para Lageado Grande - SC, Barros, Guilherme e Carvalho (2010) em Jataí - GO. Assim é necessária a adoção de medidas que visem proporcionar ambientes 
heterogêneos com a abundância de espécies nativas, além de focar na minimização de conflitos entre a urbe e a arborização.

\section{CONCLUSÕES}

O município de Godoy Moreira apresenta homogeneidade de espécies arbóreas pois através do censo realizado, foi possível observar que apenas duas espécies representam $45 \%$ da arborização de vias públicas, sendo elas Poincianella pluviosa var. peltrophoroides e Terminalia catappa que causam conflitos com os equipamentos públicos, onde se sugere a redução de seu plantio e substituição por espécies adequadas a arborização, tais como Casearia sylvestris Sw (Guaçatomba) Lafoensia pacari A.St.Hil.(Dedaleiro) e, Metrodorea nigra A.St.-Hil (Carrapateira).

Ainda, é notória a predominância de espécies exóticas e exóticas invasoras, que representa $76 \%$ da arborização de vias públicas do município e plantas inadequadas, tais com Melia azedarach e Ligustrum lucidum que além de possuírem restrições de acordo com a legislação do Paraná, ainda apresentam potencial tóxico, tornando assim inadequadas para a arborização urbana, o que demonstra a total falta de planejamento por parte dos órgãos públicos do município, fato este observado de maneira geral em grande parte das cidades brasileiras.

\section{REFERÊNCIAS}

ALBERTIN, R. M.; De ANGELIS F.; De ANGELIS NETO R.; De ANGELIS B. L. D. Diagnóstico Quali-quantitativo da Arborização Viária de Nova Esperança, Paraná, Brasil. Revista da Sociedade Brasileira de Arborização Urbana, Piracicaba - SP, v.6 n. 3, p. 128-148, 2011.

APG III, ANGIOSPERM PHYLOGENY GROUP. An update of the Angiosperm Phylogeny Group Classification for the Orders and Families of Flowering Plants: APG III. Botanical Journal of the Linnean Society, London, v. 161, p. 105-121, 2009.

BARROS, B. F. S.; GUILHERME, A. G.; CARVALHO, R. S.; Arborização Urbana Em Quadras De Diferentes Padrões Construtivos Na Cidade De Jataí. Revista Árvore, ViçosaMG, v. 34, n. 2, p. 287-295, 2010.

BIONDI, D.; PEDROSA-MACEDO, J. H. Plantas Invasoras Encontradas na Área Urbana de Curitiba (PR). Revista Floresta, Curitiba-PR, v. 38, n. 1, p. 129-144, 2008. 
BLUM, C. T; BORGO, M; SAMPAIO, A. C. F. Espécies exóticas invasoras na arborização de vias públicas de Maringá - PR. Revista Brasileira de Arborização Urbana, Piracicaba - SP, v. 3, n. 2, p. 78-97, 2008.

CADORIN, D. A.; SILVA, L. M.; HASSE, I.; BETT, C. F.; EMER, A.; OLIVEIRA, J. R. Características da arborização dos bairros Cadorin, Parzianello e La Salle em Pato Branco PR. Revista da Sociedade Brasileira de Arborização Urbana, Piracicaba - SP v. 3, n. 4, p. $40-52,2008$.

CAVIGLIONE, J. H.; KIIHL, L. R. B.; CARAMORI, P. H.; OLIVEIRA, D. Cartas climáticas do Paraná. Londrina: IAPAR, 2000.

COMPANHIA PARANAENSE DE ENERGIA (COPEL). Arborização de vias Públicas: Guia para Municípios. 2009.

EMBRAPA - EMPRESA BRASILEIRA DE AGROPECUÁRIA. Levantamento e Reconhecimento dos Solos do estado do Paraná, escala 1:250.000. Rio de Janeiro: Embrapa Solos, 2007.

EMER, A. A.; CADORIN, D. A.; SILVA, L. da; MELLO, N. A. Arborização Dos Bairros Veneza E Aeroporto Em Pato Branco - Pr. Revista da Sociedade Brasileira de Arborização Urbana, Piracicaba - SP, v. 9, n. 2, p. 88, 2014.

HOPPEN, M. I.; DIVENSI, H. F.; RIBEIRO, R. F.; CAXAMBU, M. G. Espécies Exóticas Na Arborização De Vias Públicas No Município De Farol, PR, Brasil. Revista da Sociedade Brasileira de Arborização Urbana, Piracicaba - SP, v. 9, n. 3, p. 176, 2014.

INSTITUTO BRASILEIRO DE GEOGRAFIA E ESTATÍSTICA (IBGE), 2014. Paraná Godoy Moreira. Disponível em: < http://www.cidades.ibge.gov.br/xtras/perfil.php?lang=\& codmun =410855\&search=parana |godoy-moreira >. Acesso em: 26 jun. 2015.

INSTITUTO BRASILEIRO DE GEOGRAFIA E ESTATÍSTICA (IBGE). MANUAL TÉCNICO DA VEGETAÇÃo BRASILEIRA. Séries Manuais Técnicos em Geociências. Rio de Janeiro, p. 92, 2012.

IWATA, B. F. Diagnóstico Sobre o Confronto Entre a arborização e o Planejamento Urbano no Bairro vermelha em Teresina/PI In: II Congresso de Pesquisa e Inovação da Rede Norte e Nordeste de Educação Tecnológica, 6, 2007. João Pessoa/PB. Anais. II Conep, 2007. Disponível em: www.redenet.edu.br. Acesso em: 25 de mai. 2015.

KÜSTER, L. C; STEDILLE, L. L. B; DACOREGIO, H, SILVA, A. C; HIGUCHI, P. Avaliação de riscos e procedência de espécies arbóreas nas escolas estaduais de Lages, SC. Revista de Ciências Agroveterinárias, Lages - SC, v. 11, n. 2, p. 55-67, 2012.

LISTA DE ESPÉCIES DA FLORA DO BRASIL. Jardim Botânico do Rio de Janeiro. Disponível em: <http://floradobrasil.jbrj.gov.br/>. Acesso em: 06 Jun. 2015.

MAIORANI, E.; WESOLOWSKI, J.B.; MELISINAS, V.A.P.dos S.; FABRIN, T.C.; GASQUES, L.S. Levantamento da Arborização Urbana do município de Altônia-PR. Publicatio UEPG Ciências Biológicas e da Saúde, Ponta Grossa-PR, v.18, n. 2, p. 101-108, 2012.

MARTINS, L. F. V.; ANDRADE, H. H. B. de; HANISCH, R. F.; DE ANGELIS, B. L. D.; CAXAMBU, M. G. Análise da compatibilidade da arborização viária com o ambiente construído na cidade de Luiziana, Paraná, Brasil. Revista da Sociedade Brasileira de Arborização Urbana, Piracicaba-SP, v. 6, n. 3, p. 103-127, 2011. 
NETO, E. M. de L; SOUZA, R. M. Índices De Densidade E Sombreamento Arbóreo Em Áreas Verdes Públicas De Aracaju, Sergipe. Revista da Sociedade Brasileira de Arborização Urbana, Piracicaba-SP, v. 4, n. 4, p. 47-62, 2009.

PAGLIARI, S. C; DURIGON, E. B. Arborização urbana: importância das espécies adequadas.2013. Unoesc \& Ciência - ACET, Joaçaba -SC, v. 4, n. 2, p. 139-148, 2013

PARANA, Portaria IAP n 59, de 15 de abril de 2015. Reconhece a Lista Oficial de Espécies Exóticas Invasoras para o Estado do Paraná, estabelece normas de controle e dá outras providências. Diário Oficial do Estado do Paraná. Curitiba, 2015. Disponível em: < http://www.iap.pr.gov.br/arquivos/File/Legislacao_ambiental/Legislacao_estadual/PORTARIA S/PORTARIA_IAP_125_2009_ESPECIES_EXOTICAS.pdf>

RODERJAN, C. V.; GALVÃO, F; KUNIYOSHI, Y. S.; HATSCHBACH, G. As Unidades Fitogeográficas do Paraná. Revista Ciência e Ambiente, Santa Maria-RS, v.1, n.1, p.72-95. 2002.

SOUZA, R. C.; CINTRA, D. P. Arborização viária e conflitos com equipamentos urbanos no Bairro Taquara, RJ. Revista Floresta e Ambiente, Rio de Janeiro-RJ, v. 14, n. 1, p. 45-51, 2007.

SOUZA, A. I; FERREIRA, R. A; MELLO, A. A; PLÁCIDO, D. R; SANTOS, C. Z. A; GRAÇA, D. A. S; DANTAS, J. D. M; JÚNIOR, P. P. A; BARRETO, S. S, B; DANTAS, J. D. M; PAULA, J. W. A, SILVA, T. L; GOMES, L. P. S. Diagnóstico quantitativo e qualitativo da arborização das praças de Aracaju, SE. Revista Árvore, Viçosa - MG, v. 35, n. 6, p.1253-1263, 2011.

SOUZA, F. P. A; PAIVA, A. M. S. Levantamento arbóreo do instituto federal de educação, ciência e tecnologia de São Paulo (IFSP), Campus São Paulo. Revista Brasileira de Arborização Urbana, Piracicaba - SP, v. 9, n. 2, p.77-86, 2014. 